\title{
МЕТОДИКА ТЕРМОЛЮМИНЕСЦЕНТНОГО ДАТИРОВАНИЯ В ГЕОЛОГИИ
}

За последние годы метод термолюминесцентного (ТЛ) датирования нашел широкое применение в археологии. В предыдущей работе (Хютт, Раукас, 1977) нами были проанализированы публикации,' где описывались наиболее известные в археологии методики, а также показана несостоятельность используемой рядом лабораторий СССР методики ТЛ датирования в геологии (Шелкопляс, 1974) в связи с отсутствием физической разработки вопроса.

В настоящем сообщении основное внимание уделено физическому аспекту проблемы ТЛ датирования в геологии и предложена новая методика, которая, на наш взгляд, весьма перспективна в практическом использовании.

Проведенное изучение ТЛ и дозиметрических свойств кварца (Хютт и др., 1977) показало, что этот минерал под действием природных радиоактивных примесей $\mathrm{U}$, Th и ${ }^{40} \mathrm{~K}$ способен запасать и сохранять информацию в виде светосуммы в течение $10^{3}-5 \cdot 10^{6}$ лет, что в принципе позволяет определять возраст осадочных отложений во всем интервале четвертичного периода при условии полного стирания прогенетической светосуммы кварца.

Возраст $T$ (в годах) вычисляется по формуле

$$
T=\frac{\text { ЕТЛ } \cdot D_{\mathrm{a}}}{\text { ИТЛ } \cdot D_{\mathrm{r}}},
$$

где ЕТЛ и ИТЛ - естественная и индуцированная эталонным источником ТЛ кварца; $D_{\text {a и }} D_{\Gamma}$ - аккумулированная и годичная дозы, рад.

Данная методика основана на методике крупных включений, наиболее широко используемой в археологии (Fleming, 1970). Ниже излагаются основные этапы работы.

\section{Методика ТЛ датирования}

Выделени ев карцевого об разца. Экстрагирование кварца крупностью $100-140$ мкм и фазовой чистотой 98-99\% производили согласно методике, описанной ранее (Хютт и др., 1977). Дополнительная препаративная обработка образцов кварца была вызвана следующими соображениями. Как известно в природных цепочках распада U, Th содержатся изотопы, являюшиеся $\alpha-, \beta-, \gamma$-источниками. Кроме того, источником возбуждения служит ${ }^{40} \mathrm{~K}$, в основном, излучатель $\beta$-частиц. 
В кварце пробег $\alpha$-частиц составляет $\sim 20$ мкм, $\beta$-частиц $\sim 2$ мм, проникающая способность $\gamma$-квантов $\sim 5$ см (Mark и др., 1971). Таким образом, для выбранного нами размера частиц $100-140$ мкм $\alpha$-облучение играет роль поверхностной активации. Известно также (Tite, 1966), что кварц обладает селективными дозиметрическими свойствами: чувствительность ТЛ к $\alpha$-облучению примерно в 10 раз слабее ее чувствительности к $\beta$-облучению. Следовательно, вклад в ТЛ $\alpha$-компоненты гораздо менее существен, чем $(\beta+\gamma)$-компонент. Кроме того, обнаружено (Шехмаметьев, Пор, 1975), что $\alpha$-облучение высвечивает светосумму, запасенную кварцем под влиянием $\gamma$-дозы. Эти соображения диктуют целесообразность избавления от вклада в ТЛ $\alpha$-дозы. Препаративная процедура состоит в обработке кварцевой фракции 40\%-ной плавиковой кислотой (в течение часа растворяется верхний слой толщиной 220 мкм). Схема получения кварцевого образца для ТЛ датирования выглядит следующим образом: валовая проба $\rightarrow$ мокрый ситовый анализ $(100-140$ мкм $) \rightarrow$ обработка в течение 10 мин смесью $\mathrm{HF}+\mathrm{HCl}($ по $30 \mu \Omega)+\mathrm{H}_{2} \mathrm{O}(300 \mu \Omega) \rightarrow$ минеральная сепарация кварцевой фракции $D=2,62-2,67 \mathrm{a}_{\mathrm{c}} \mathrm{s}^{3} \rightarrow$ обработка в течение 1 ч $40 \%$-ной HF. Bce препаративные процедуры проводились при ослабленном красном свете во избежание высвечивания ЕТЛ.

Ап п а а тур а реги т р ц и и ТЛ. Блок-схема установки показана на рис. 1. Основной частью ее является герметичный зеркальный криостат. Образец кварца $(\approx 40$ мг) помещается на металлическую подложку и нагревается находящейся извне трубчатой печью со скоростью 0,3 град/сек. Сигнал ТЛ фиксируется ФЭУ-97, перед которым установлен жидкостный фильтр с насыщенным раствором $\mathrm{CuSO}_{4}$ для отрезания фонового свечения. Нагрев от 230 до $700 \mathrm{~K}$ производится равномерно с помощью специально разработанного автоматического устройства. Отметки температуры, контролируемые термопарой, автоматически наносятся на кривую ТЛ. Процесс ТЛ осуществляется в атмосфере азота высокой степени чистоты (кислорода менее 0,001\%) во избежание «ложной» компоненты ТЛ (Хютт и др., 1977). Перед напуском азота система вакуумируется.

Определение акку м ули ров анной доы. ИТЛ возбуждалась $\gamma$-пушкой ${ }^{60} \mathrm{Co}$, мощность экспозиционной дозы составляла 441 p/сек. Поскольку характер заполнения ловушек носителями зависит от типа возбудителя, то при ис-

Рис. 1. Блок-схема установки для измерения ТЛ: $1-$ форвакуумный насос, 2 - баллон со сжатым азотом, 3 - блок нагрева, 4 - блок управления скоростью нагрева, $5-$ блок высокого напряжения, $6-$-усилитель постоянного тока, 7 блок автоматического переключения сигналов ТЛ и температуры, 8 - фотоэлектронный умножитель, 9 - криостат, 10 - самописец.

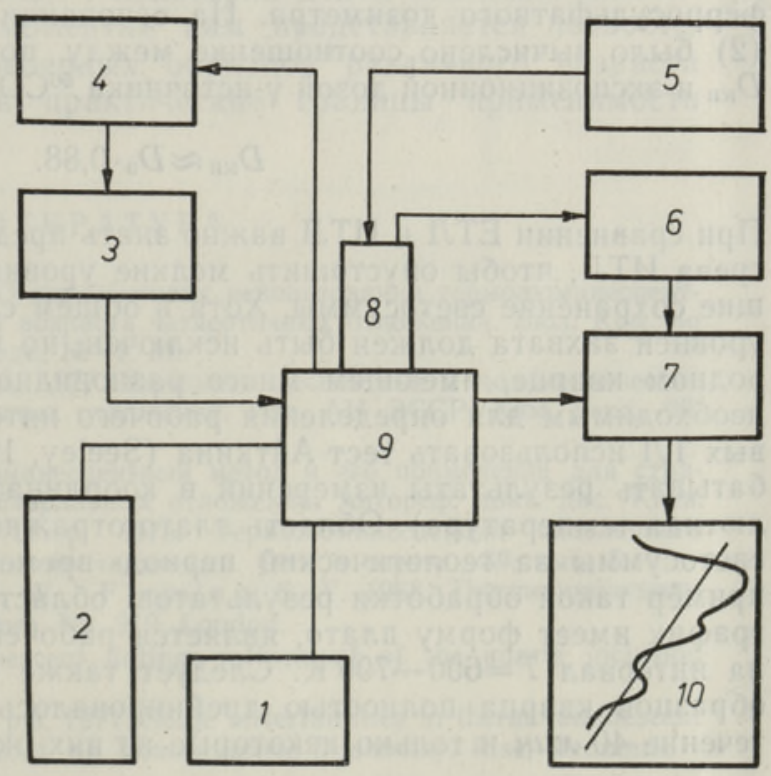

3 ENSV TA Toimetised K* G -41977 


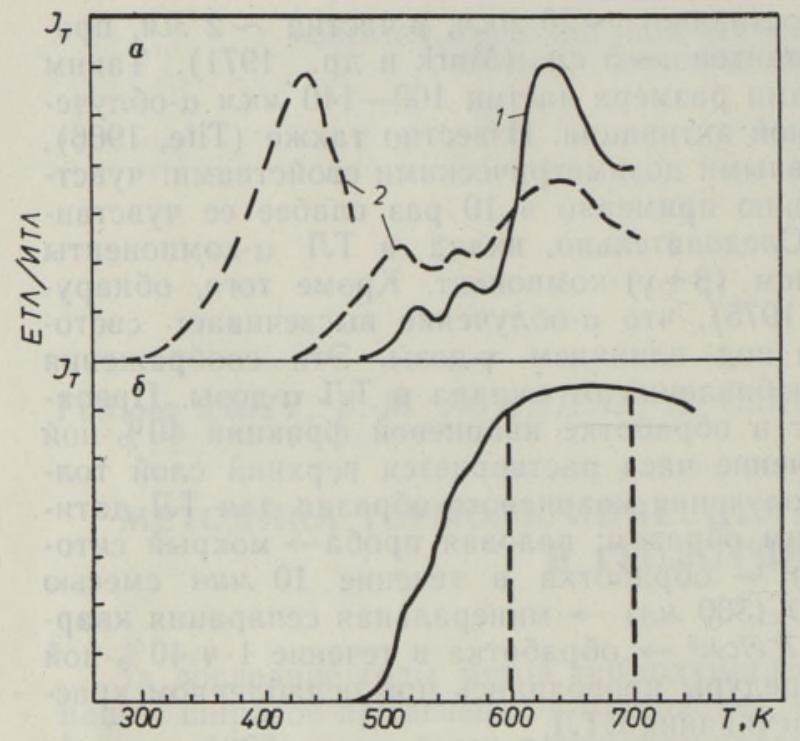

кусственном возбуждении необходимо более полное моделирование природных условий: $\gamma$-возбуждение от источника ${ }^{60} \mathrm{Co}$ (рис. 2,a) вполне удовлетворительно выполняет эту функцию (при рентгеновском возбуждении картина существенно иная). Сравнение ЕТЛ и ИТЛ, индуцированной от источника радиации известной мощности, позволяет вычислить аккумули-

Рис. 2, а. Моделирование природных процессов возбуждения при $\gamma$-облучении ${ }^{60} \mathrm{Co}$ : ЕТЛ $(l)$, ИТЛ (2); б - рабочий диапазон температур для определения возраста образца.

рованную дозу. Известную трудность для экспериментаторов представляет переход от экспозиционной дозы к поглощенной. Нами были сделаны соответствующие вычисления по формуле

$$
D_{\text {вещ. }}=D_{\ni} \cdot 0,88 \frac{\mu / \varrho_{\text {вещ. }}}{\mu / \varrho_{\text {возд. }}} \text {, }
$$

где $D_{\text {вещ. }}$ - доза, поглощенная веществом, $p a \partial ; D_{3}-$ экспозиционная

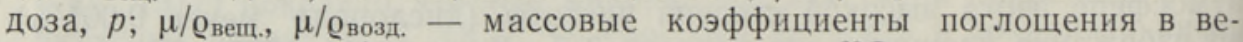
ществе и воздухе. $\mu / \varrho$ кварца для $\gamma$-источника ${ }^{60} \mathrm{Co}$ вычислено нами и равно $0,0260 c \mu^{2} / 2 ; \mu / 0$ воздуха равно $0,0267 c \mu^{2} / 2 ; \beta$-излучение, генерируемое ${ }^{60} \mathrm{Co}$, поглощалось фильтром. $D_{2}$ была получена с помощью ферросульфатного дозиметра. На основании этих данных из формулы (2) было вычислено соотношение между поглощенной кварцем дозой

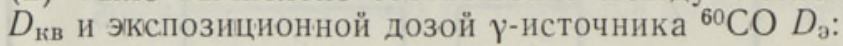

$$
D_{\mathrm{\kappa B}} \approx D_{3} \cdot 0,88
$$

При сравнении ЕТЛ и ИТЛ важно знать пределы предварительного прогрева ИТЛ, чтобы опустошить мелкие уровни захвата, не обеспечивающие сохранение светосуммы. Хотя в общем случае фединг для глубоких уровней захвата должен быть исключен, но поскольку речь идет о природном кварце, имеющем много разновидностей, нам представляется необходимым для определения рабочего интервала температур на кривых ТЛ использовать тест Айткина (Seeley, 1975), т. е. графически обрабатывать результаты измерений в координатах ЕТЛ/ИТЛ, $T$ К (абсолютная температура). Область плато отражает стабильность запасенной светосуммы за геологический период времени. На рис. 2,б приведен пример такой обработки результатов: область температур, при которых график имеет форму плато, является рабочей и приходится в основном на интервал $T=600-700 \mathrm{~K}$. Следует также отметить, что большинство образцов кварца полностью дрейнировалось при температуре $820 \mathrm{~K}$ в течение 40 мин и только некоторые из них оказались сенсибилизирован- 
ными при последующем облучении. Во избежание возможных ошибок лучше, по-видимому, воздействовать на невысвеченный образец индуцированной дозой, величина которой должна быть близка к величине аккумулированной дозы.

О пределение годичной дозы. Основными источниками активации природного кварца, как было указано выше, являются ${ }^{40} \mathrm{~K}$ и $(\beta+\gamma)$-активные изотопы из природных рядов $\mathrm{U}$ и $\mathrm{Th}(\alpha$-активированная поверхность зерен кварца растворялась $\mathrm{HF}$ ). Содержание ${ }^{40} \mathrm{~K}$ определялось методом пламенной фотометрии, а содержания U и $\mathrm{Th}-$ $\gamma$-спектрометрически и химически. Наиболее перспективным нам представляется метод $\alpha$-спектрометрии с предварительным радиохимическим разделением U и Th. Эта методика в настоящее время внедряется в нашей лаборатории.

При измерении концентраций радиоактивных изотопов особое внимание должно уделяться получению среднего образца и проведению параллельных измерений, чтобы уменьшить вероятность случайных показаний. При переходе от концентраций радиоактивных изотопов к поглощенной дозе нами были использованы экспериментально определенные коэффициенты пересчета (Aitken и др., 1968): 1\% K $\rightarrow 0,113$ рад; $10^{-4} \% \mathrm{U} \rightarrow 0,025$ рад; $10^{-4} \%$ Th $\rightarrow 0,00742$ рад (учитывали лишь $(\beta+\gamma)$-компоненты доз). Теоретические расчеты показали хорошее совпадение с эмпирическими данными.

Следует отметить, что большинство исследованных нами образцов содержали U и Th в количествах, меньших кларковых. Поэтому вклад в общую $(\beta+\gamma)$-дозу от ${ }^{40} \mathrm{~K}$ у большей части образцов составляет $65-$ $85 \%$. Зная общее содержание калия в образце и измеряя $\beta$-активность на двухканальном $\beta$-спектрометре, можно производить предварительный отбор образцов, вклад которых в суммарную $(\beta+\gamma)$-дозу от U и Th относительно невелик. Этот вывод нам представляется важным и с точки зрения достоверности получаемых датировок, ибо для указанных случаев ошибка, вызванная неконтролируемыми процессами миграции $\mathrm{U}$ и Th, будет невелика.

Полученные нами датировки оказались вполне разумными с геологической точки зрения. Но в связи с некоторой долей сомнения относительно определенности «нуль-момента» нам представляется первостепенной задачей датирование реперных образцов различного возраста и генезиса, чтобы установить практические границы применимости метода.

\section{Л И ТЕ Р А Т У Р А}

Х ют т Г. И., Р ау к а с А. В. 1977. О перспективах использования термолюминесцентного метода для определения возраста четвертичных отложений. Бюл. Ком. по изучению четвертичного периода, № 47. М.

Х ют т Г., В а р е с К., С м и р н в А. 1977. Термолюминесцентные и дозиметрические свойства кварца из четвертичных отложений. Изв. АН ЭССР, Хим. Геол., 26, № 4 .

Шелкопляс В. Н. 1974. Термолюминесцентный метод и его применение для стратиграфии плейстоценовых субаэральных отложений. Автореф. докт. дис. Киев.

Ш ехм а м ет ве в Р. И., Г а бор П о р. 1975. Термолюминесценция плавленного кварца, облученного заряженными частицами. Опт. и спектр., 39, вып. 5.

A itken, M. Y., Z i m m e r m a n, D. W., Flem ing, S. Y. 1968. Thermoluminescent dating of ancient pottery, Nature, No. 219, London.

Fleming, S. Y. 1970. Thermoluminescent dating: refinement of the quartz inclusion method. Archaeometry, No. 2.

M a r k, C., Han and Elizabeth K. R a l p h, 1971. Some uncertainities in thermoluminescent dating. Proc. of 3rd Intern. Conf. on Luminescence Dosimetry. Risø, Denmark. 
Se eley, M. A. 1975. Thermoluminescent dating in its application in Archaeology. J. of Archaeol. Sci., No. 2.

Tite, M. S. 1966. Thermoluminescent dating of ancient ceramics. Archaeometry, No. 9.

Ннститут геологии

Академии наук Эстонской ССР
Поступила в редакцию $27 / \mathrm{V} 1976$

Galina HOTT, J.-M. PUNNING, A. SMIRNOV

\section{GEOLOOGILISTE OBJEKTIDE DATEERIMINE TERMOLUMINESTSENTSIMEETODIL}

Antakse kvartsi termoluminestsentsispektrite ja dosimeetriliste omaduste uurimisele tuginev ülevaade geoloogiliste setete vanuse määramiseks kasutatavast termoluminest. sentsimeetodist.

Uuritavast proovist eraldati sõelanalüüsi ja mineraloogilise separeerimise abil kvartsi fraktsioon 100-140 $\mu$ ning mõödeti selle looduslik termoluminestsents (LTL) puhtas lämmastikus. Preparaati ergastati $\gamma$-allikaga $\left({ }^{60} \mathrm{Co}\right)$ ning indutseeritud termoluminestsents (ITL) võrdlemisel looduslikuga saadi kvartsil akumuleerunud summaarne doos $\left(D_{\mathrm{a}}\right)$ Proovi radioaktiivsete isotoopide $\left(\mathrm{U}, \mathrm{Th},{ }^{40} \mathrm{~K}\right)$ sisalduse määramise kaudu leiti aastane akumuleerunud doos $\left(D_{\mathrm{r}}\right)$. Et $\alpha$-osakeste mõju elimineeriti kristallide välise osa lahusta mise teel, tuleb arvestada ainult $(\beta+\gamma)$ kiirguse mõjul toimunud aktiviseerumist. Saadud andmed võimaldavad leida proovi ladestumisest möödunud ajavahemiku $T$ :

$$
T=\frac{\operatorname{LTL} D_{\mathrm{a}}}{\operatorname{ITL} D_{\mathrm{r}}} .
$$

Esialgsed uurimused näitavad, et termoluminestsentsimeetod on kasutatav $10^{3}-5 \cdot 10^{4}$ aastat tagasi ladestunud setete dateerimiseks. Edasised uurimused peavad kinnitame meetodi usaldusväärsust ja välja selgitama piiravad tingimused.

\section{Galina HOTT, J.-M. PUNNING, A. SMIRNOV}

\section{THERMOLUMINESCENT DATING IN ITS APPLICATION TO GEOLOGY}

On the grounds of the thermoluminescent and dosimetrical peculiarities of quartz, brief account on the thermoluminescent method, used for dating geological deposits, presented.

The fraction of quartz (the size of granules from 100 to $140 \mu$ ) is secluded from the sample by sieve analyses and mineralogical separation. The natural luminescence of the preparation is measured in the atmosphere of pure nitrogen. The preparation is stimulated with $\gamma$-source $\left({ }^{60} \mathrm{Co}\right)$, and the total dose $\left(D_{\mathrm{a}}\right)$, accumulated by quartz, is determined comparing the induced thermoluminescence (ITL) with the natural one (NTL). On the ground of the content of the radioactive isotopes (U, Th, ${ }^{40} \mathrm{~K}$ ) in the sample, the annual accumulated dose $\left(D_{\mathrm{r}}\right)$ is determined. As the action of the $\alpha$-particles is eliminated by dissolving the external parts of the crystals, one should only consider the activation caused by $(\beta+\gamma)$-radiation. The obtained data enable to determine the time that has passed since the deposition of the sample.

$$
T=\frac{\mathrm{NTL} \cdot D_{\mathrm{a}}}{\mathrm{ITL} \cdot D_{\mathrm{r}}}
$$

The supposed interval of the dating is $10^{3}-5 \cdot 10^{6}$ years. Further investigations have to prove the reliability of the method and elucidate the restricting conditions. 